\title{
Interval-valued Fuzzy Quasi-ideals in a Semigroups
}

\author{
Sang-Mok Kim ${ }^{1}$, Kul Hur ${ }^{2}$, Minseok Cheong $^{3}$, and Gab-Byung Chae ${ }^{4} \dagger$ \\ 1 Division of General Education - Mathematics, Kwangwoon University, Seoul 139-701, Korea \\ 2 Division of Mathematics and Informational Statistics, and Nanoscale Science and Technology Institute, \\ Wonkwang University, Jeollabukdo, Korea \\ 3 GyeongGi Science High School for the gifted, Gyeonggido, Korea \\ 4 Division of Mathematics and Informational Statistics, and Institute of Natural Basic Sciences, Wonkwang \\ University, Iksan, Jeollabukdo, Korea
}

\begin{abstract}
We initiate the study of interval-valued fuzzy quasi-ideal of a semigroup. In Section 2, we list some basic definitions in the later sections. In Section 3, we investigate interval-valued fuzzy subsemigroups and in Section 4, we define intervalvalued fuzzy quasi-ideals and establish some of their basic properties. In Section 5, we obtain characterizations of regular and intraregular semigroups using the machinery developed in the preceding sections.
\end{abstract}

Key Words: interval-valued fuzzy set, interval-valued fuzzy left(right) ideal, interval-valued fuzzy bi-ideal, interval-valued fuzzy quasi-ideal, regular semigroup, intraregular semigroup

\section{Introduction}

The theory of fuzzy sets proposed by Zadeh [11] in 1965 has achieved a great success in various fields. Since then, Ahsan and Latif [1] investigated fuzzy quasi-ideals in a semigroup. With the research of fuzzy sets, in 1975, Zadeh [12] introduced the notion of interval-valued fuzzy sets as a generalization of fuzzy sets. After then, Biswas [3] applied it to group theory, Mondal and Samanto [9] to topology. Recently, Kang and Hur [6] studied interval-valued fuzzy subgroups and subrings and Choi et al [5] introduced the concept of interval-valued smooth topological spaces and investigated some of its properties. In particular, Cheong and Hur [4] studied interval-valued fuzzy generalized bi-ideals of a semigroup, and Lee et al [8] investigated interval-valued fuzzy ideals and bi-ideals in a semigroup.

In this paper, we initiate the study of interval-valued fuzzy quasi-ideal of a semigroup. In Section 2, we list some basic definitions in the later sections. In Section 3, we investigate interval-valued fuzzy subsemigroups and in Section 4, we define interval-valued fuzzy quasi-ideals and establish some of their basic properties. In Section 5, we obtain characterizations of regular and intraregular semi-

Manuscript received Dec. 25, 2011; revised Jun. 18, 2012; accepted Jun. 29, 2012.

This paper was supported by Wonkwang University in 2012.

†Corresponding Author : rivendell@wonkwang.ac.kr

(c) The Korean Institute of Intelligent Systems. All rights reserved. groups using the machinery developed in the preceding sections.

\section{Preliminaries}

We will list some concepts and one result needed in the later sections.

Throughout this paper, we will denote the unit interval $[0,1]$ as $I$ and for an ordinary subset of a set $X$, we will denote the characteristic function of $A$ as $\chi_{A}$.

Let $D(I)$ be the set of all closed subintervals of the unit interval $I=[0,1]$. The elements of $D(I)$ are generally denoted by capital letters $M, N, \cdots$, and note that $M=\left[M^{L}, M^{U}\right]$, where $M^{L}$ and $M^{U}$ are the lower and the upper end points respectively. Especially, we denoted, $\tilde{0}=[0,0], \tilde{1}=[1,1]$, and $\mathbf{a}=[a, a]$ for every $a \in(0,1)$. We also note that $N^{(i)}$ $\left.N^{U}\right)$.

(ii) $(\forall M, N \in D(I))\left(M \leq N \Leftrightarrow M^{L} \leq N^{L}, M^{U} \leq\right.$ For every $M \in D(I)$, the complement of $M$, denoted by $M^{c}$, is defined by $M^{c}=1-M=\left[1-M^{U}, 1-M^{L}\right]$.

Definition 2.1. [12]. A mapping $A: X \rightarrow D(I)$ is called an interval-valued fuzzy set (in short, IVS) in $X$, denoted by $A=\left[A^{L}, A^{U}\right]$, if $A^{L}, A^{U} \in I^{X}$ such that $A^{L} \leq A^{U}$, i.e., $A^{L}(x) \leq A^{U}(x)$ for each $x \in X$, where $A^{L}(x)$ [resp. $A^{U}(x)$ ] is called the lower[resp. upper] end point of $x$ 
to $A$. For any $[a, b] \in D(I)$, the interval-valued fuzzy set $A$ in $X$ defined by $A(x)=\left[A^{L}(x), A^{U}(x)\right]=[a, b]$ for each $x \in X$ is denoted by $[\widetilde{a, b}]$ and if $a=b$, then the IVS $\widetilde{[a, b]}$ is denoted by simply $\widetilde{a}$. In particular, $\tilde{0}$ and $\tilde{1}$ denote the interval-valued fuzzy empty set and the interval-valued fuzzy whole set in $X$, respectively. We will denote the set of all IVSs in $X$ as $D(I)^{X}$. It is clear that set $A=\left[A^{L}, A^{U}\right] \in D(I)^{X}$ for each $A \in I^{X}$.

Definition 2.2. $[9,12]$. Let $A, B \in D(I)^{X}$ and let $\left\{A_{\alpha}\right\}_{\alpha \in \Gamma} \subset D(I)^{X}$. Then:
(a) $A \subset B$ iff $A^{L} \leq B^{L}$ and $A^{U} \leq B^{U}$.
(b) $A=B$ iff $A \subset B$ and $B \subset A$.
(c) $A^{c}=\left[1-A^{U}, 1-A^{L}\right]$.
(d) $A \cup B=\left[A^{L} \vee B^{L}, A^{U} \vee B^{U}\right]$.
$(d)^{\prime} \bigcup_{\alpha \in \Gamma} A_{\alpha}=\left[\bigvee_{\alpha \in \Gamma} A_{\alpha}^{L}, \bigvee_{\alpha \in \Gamma} A_{\alpha}^{U}\right]$
(e) $A \cap B=\left[A^{L} \wedge B^{L}, A^{U} \wedge B^{U}\right]$.
$(e)^{\prime} \bigcap_{\alpha \in \Gamma} A_{\alpha}=\left[\bigwedge_{\alpha \in \Gamma} A_{\alpha}^{L}, \bigwedge_{\alpha \in \Gamma} A_{\alpha}^{U}\right]$.

\section{Interval-valued fuzzy subsemigroups}

Definition 3.1. [6]. Let $(X, \cdot)$ be a groupoid and let $A, B \in$ $D(I)^{X}$. Then the interval-valued fuzzy product of $A$ and $B$, denoted by $A \circ B$, is an IVS in $X$ defined as follows : For each $x \in X$,

$$
(A \circ B)(x)= \begin{cases}{[a, b],} & \text { if } y z=x ; \\ {[0,0],} & \text { otherwise. }\end{cases}
$$

where $a=\bigvee_{y z=x}\left(A^{L}(y) \wedge B^{L}(z)\right), b=\bigvee_{y z=x}\left(A^{U}(y) \wedge\right.$ $\left.B^{U}(z)\right)$. It is clear that for any $A, B, C \in D(I)^{X}$, if $B \subset$ $C$, then $A \circ B \subset A \circ C$ and $B \circ A \subset C \circ A$.

Result 3.A. [6, Proposition 3.3]. Let $(S, \cdot)$ be a groupoid. (a) If "." is associative[resp. commutative], then so is $\circ$ in $D(I)^{S}$.

(b) If "." has an identity $e \in S$, then $e_{1} \in I V F_{p}(X)$ is an identity of $\circ$ in $D(I)^{S}$.

Proposition 3.2. Let $S$ be a groupoid and let $A, B, C \in$ $D(I)^{S}$. Then

(a) $A \circ(B \cup C)=(A \circ B) \cup(A \circ C),(B \cup C) \circ A=$ $(B \circ A) \cup(C \circ A)$.

(b) $A \circ(B \cap C) \subset(A \circ B) \cap(A \circ C),(B \cap C) \circ A \subset$ $(B \circ A) \cap(C \circ A)$.

Proof. (a) Let $x \in S$. Suppose $x$ is not expressible as $x=y z$. Then clearly $(A \circ(B \cup C))(x)=\tilde{0}=((A \circ B) \cup$ $(A \circ C))(x)$. Suppose $x$ is expressible as $x=y z$. Then

$$
(A \circ(B \cup C))^{L}(x)=\bigvee_{x=y z}\left(A^{L}(y) \wedge(B \cup C)^{L}(z)\right.
$$

$$
\begin{aligned}
& =\bigvee_{x=y z}\left(A^{L}(y) \wedge\left(B^{L}(z) \vee C^{L}(z)\right)\right. \\
& =\bigvee\left(\left(A^{L}(y) \wedge B^{L}(z)\right) \vee\left(A^{L}(y) \wedge C^{L}(z)\right)\right) \\
& =\bigvee\left(A^{L}(y) \wedge B^{L}(z)\right) \vee \bigvee_{x=y z}\left(A^{L}(y) \wedge C^{L}(z)\right) \\
& =y z \\
& =(A \circ B)^{L}(x) \vee(A \circ C)^{L}(x) \\
& =((A \circ B) \cup(A \circ C))^{L}(x) .
\end{aligned}
$$

Thus $A \circ(B \cup C)=(A \circ B) \cup(A \circ C)$. By the similar arguments, we have $(B \cup C) \circ A=(B \circ A) \cup(C \circ A)$.

(b) Let $x \in S$. Suppose $x$ is not expressible as $x=y z$. Then clearly $(A \circ(B \cap C))(x)=\tilde{0}=((A \circ B) \cap(A \circ C))(x)$. Suppose $x$ is expressible as $x=y z$. Then

$$
\begin{aligned}
(A & \circ(B \cap C))^{L}(x)=\bigvee_{x=y z}\left(A^{L}(y) \wedge(B \cap C)^{L}(z)\right) \\
& =\bigvee_{x=y z}\left(A^{L}(y) \wedge\left(B^{L}(z) \wedge C^{L}(z)\right)\right) \\
& =\bigvee_{x=y z}\left(\left(A^{L}(y) \wedge B^{L}(z)\right) \wedge\left(A^{L}(y) \wedge C^{L}(z)\right)\right) \\
& \leq \bigvee_{x=y z}\left(A^{L}(y) \wedge B^{L}(z)\right) \wedge \bigvee_{x=y z}\left(A^{L}(y) \wedge C^{L}(z)\right) \\
& =(A \circ B)^{L}(x) \wedge(A \circ C)^{L}(x) \\
& =((A \circ B) \cap(A \circ C))^{L}(x) .
\end{aligned}
$$

Similarly, we have that $(A \circ(B \cap C))^{U}(x) \leq((A \circ B) \cap$ $(A \circ C))^{U}(x)$. Thus $A \circ(B \cap C) \subset(A \circ B) \cap(A \circ C)$. By the similar arguments, we have $(B \cap C) \circ A \subset(B \circ A) \cap(C \circ A)$. This completes the proof.

Let $S$ be a semigroup. By a subsemigroup of $S$ we mean a non-empty subset of $A$ such that $A^{2} \subset A$ and by a left [resp. right] ideal of $S$ we mean a non-empty subset $A$ of $S$ such that $S A \subset A$ [resp. $A S \subset A$ ]. By two-sided ideal or simply ideal we mean a subset $A$ of $S$ which is both a left and a right ideal of $S$. We will denote the set of all left ideals[resp. right ideals and ideals] of $S$ as $\mathrm{LI}(\mathrm{S})[$ resp. $\mathrm{RI}(\mathrm{S})$ and $\mathrm{I}(\mathrm{S})]$.

Definition 3.3. [8]. Let $S$ be a semigroup and let $\widetilde{0} \neq A \in$ $D(I)^{S}$. Then $A$ is called an:

(i) interval-valued fuzzy semigroup (in short, $I V S G$ ) of $S$ if $A^{L}(x y) \geq A^{L}(x) \wedge A^{L}(y)$ and $A^{U}(x y) \geq A^{U}(x) \wedge$ $A^{U}(y)$ for any $x, y \in S$.

(ii) interval-valued fuzzy left ideal(in short, $I V L I$ ) of $S$ if $A^{L}(x y) \geq A^{L}(y)$ and $A^{U}(x y) \geq A^{U}(y)$ for any $x, y \in S$.

(iii) interval-valued fuzzy right ideal(in short, $I V R I$ ) of $S$ if $A^{L}(x y) \geq A^{L}(x)$ and $A^{U}(x y) \geq A^{U}(x)$ for any $x, y \in S$.

(iv) interval-valued fuzzy(two-sided) ideal (in short, $I V I)$ of $S$ if it is both an IVLI and an IVRI of $S$. 
We will denote the set of all IVSGs [resp.IVLIs, IVRIs and IVIs] of $S$ as IVSG(S) [resp.IVLI(S), IVRI(S) and IVI(S)]. It is clear that $A \in \operatorname{IVI}(\mathrm{S})$ if and only if $A^{L}(x y) \geq A^{L}(x) \wedge A^{L}(y)$ and $A^{U}(x y) \geq A^{U}(x) \wedge A^{U}(y)$ for any $x, y \in S$, and if $A \in \operatorname{IVLI}(\mathrm{S})[$ resp. IVRI(S) and IVI(S)], then $A \in \operatorname{IVSG}(\mathrm{S})$.

The following is the immediate result of Definitions 3.1 and 3.3(i).

Proposition 3.4. Let $S$ be a semigroup and let $\tilde{0} \neq A \in$ $D(I)^{S}$. Then $A \in \operatorname{IVSG}(\mathrm{S})$ if and only if $A \circ A \subset A$.

Result 3.B. [3, Proposition 3.4]. Let $A$ be a non-empty subset of a semigroup $S$.

(a) $A$ is a subsemigroup of $S$ if and only if $\left[\chi_{A}, \chi_{A}\right] \in$ IVSG(S).

(b) $A \in \operatorname{LI}(\mathrm{S})[$ resp. $\mathrm{RI}(\mathrm{S})$ and $\mathrm{I}(\mathrm{S})]$ if and only if $\left[\chi_{A}, \chi_{A}\right] \in \operatorname{IVLI}(\mathrm{S})$ [resp. IVRI(S) and $\left.\operatorname{IVI}(\mathrm{S})\right]$.

Result 3.C. [4, Lemmas 2.3 and 2.4]. Let $S$ be a semigroup and let $\tilde{0} \neq A \in D(I)^{S}$. Then $A \in \operatorname{IVLI}(\mathrm{S})[$ resp. $\operatorname{IVRI}(\mathrm{S})]$ if and only if $\tilde{1} \circ A \subset A[$ resp. $A \circ \tilde{1} \subset A]$.

Proposition 3.5. Let $S$ be a semigroup and let $A, B, C \in$ $D(I)^{S}$. If $A \subset B$, then $A \circ C \subset B \circ C$ and $C \circ A \subset C \circ B$.

Proof. Let $x \in S$. Suppose $x$ is not expressible as $x=y z$. Then clearly $(A \circ C)(x)=\tilde{0}=(B \circ C)(x)$. Suppose $x$ is expressible as $x=y z$. Then

$$
\begin{aligned}
(A \circ C)^{L}(x) & =\bigvee_{x=y z}\left(A^{L}(y) \wedge C^{L}(z)\right) \\
& \leq \bigvee_{x=y z}\left(B^{L}(y) \wedge C^{L}(z)\right)
\end{aligned}
$$

(Since $A \subset B$ )

$$
=(B \circ C)^{L}(x) .
$$

Similarly, we have that $(A \circ C)^{U}(x) \leq(B \circ C)^{U}(x)$. Hence $A \circ C \subset B \circ C$. By the similar arguments, we have $C \circ A \subset$ $C \circ B$. This completes the proof.

Proposition 3.6. Let $S$ be a semigroup and $\tilde{0} \neq A \in$ $D(I)^{S}$. Then $\tilde{1} \circ A \in \operatorname{IVLI}(\mathrm{S})[$ resp. $A \circ \tilde{1} \in \operatorname{IVRI}(\mathrm{S})]$.

Proof. $\tilde{1} \circ(\tilde{1} \circ A)=(\tilde{1} \circ \tilde{1}) \circ A \subset \tilde{1} \circ A$, by Results $3 . A$ and Proposition 3.5, respectively. Hence, by Result 3.C, $\tilde{1} \circ A \in$ $\operatorname{IVLI}(\mathrm{S})$. Similarly, we can see that $A \circ \tilde{1} \in \operatorname{IVRI(S)}$. This completes the proof.

Proposition 3.7. Let $S$ be a semigroup and $\tilde{0} \neq A \in$ $D(I)^{S}$. Then $A \cup(\tilde{1} \circ A) \in \operatorname{IVLI}(\mathrm{S})[$ resp. $A \cup(A \circ \tilde{1}) \in$ IVRI(S)].
Proof.

$$
\begin{aligned}
\tilde{1} \circ & (A \cup(\tilde{1} \circ A)) \\
= & (\tilde{1} \circ A) \cup(\tilde{1} \circ(\tilde{1} \circ A)) \\
& \quad(\text { By Proposition } 3.2(a)) \\
= & (\tilde{1} \circ A) \cup(\tilde{1} \circ \tilde{1} \circ A) \subset(\tilde{1} \circ A) \cup(\tilde{1} \circ A) \\
& \quad(\text { By Result 3.A }) \\
= & \tilde{1} \circ A \subset A \cup(\tilde{1} \circ A) .
\end{aligned}
$$

Hence, by Result 3. C, $A \cup(\tilde{1} \circ A) \in \operatorname{IVLI}(\mathrm{S})$. By the similar arguments, we can see that $A \cup(A \circ \tilde{1}) \in \operatorname{IVRI}(\mathrm{S})$. This completes the proof.

Proposition 3.8. Let $S$ be a semigroup and let $\tilde{0} \neq A \in$ $D(I)^{S}$. If $A \in \operatorname{IVRI}(\mathrm{S})[$ resp. IVLI(S)], then $A \cup(\tilde{1} \circ A) \in$ $\operatorname{IVLI}(\mathrm{S})[$ resp. $A \cup(A \circ \tilde{1})$ is an IVI of $S$.

Proof. Suppose $A \in \operatorname{IVRI}(\mathrm{S})$.

$$
\begin{aligned}
(A \cup(\tilde{1} \circ A)) \circ \tilde{1} & \\
= & (A \circ \tilde{1}) \cup((\tilde{1} \circ A)) \circ \tilde{1} \\
& \quad(\text { By Proposition 3.2 }(a)) \\
= & (A \circ \tilde{1}) \cup(\tilde{1} \circ(A \circ \tilde{1})) \\
& \quad(\text { By Result 3.A }) \\
& \subset(A \cup(\tilde{1} \circ A)
\end{aligned}
$$

(By Result 3.C and Proposition 3.5)

Thus, by Result 3.C, $A \cup(\tilde{1} \circ A) \in \mathrm{IVRI}(\mathrm{S})$. From Proposition 3.7, it is clear that $(A \cup(\tilde{1} \circ A)) \in \operatorname{IVLI}(\mathrm{S})$. So $(A \cup(\tilde{1} \circ A)) \in \operatorname{IVI}(\mathrm{S})$. Similarly, we can see that if $A \in$ $\operatorname{IVLI}(\mathrm{S})$, then $(A \cup(A \circ \tilde{1})) \in \operatorname{IVI}(\mathrm{S})$. This completes the proof.

\section{Interval-valued fuzzy quasi-ideals}

A nonempty subset $A$ of a semigroup $S$ is called a quasi-ideal of $S$ (See [10]) if $A S \cap S A \subset A$. We will denote the set of all quasi-ideals of $S$ as QI(S).

Definition 4.1. Let $S$ be a semigroup and let $\tilde{0} \neq A \in$ $D(I)^{S}$. Then $A$ is called an interval-valued fuzzy quasiideal(in short, $I V Q I)$ of $S$ if $(\tilde{1} \circ A) \cap(A \circ \tilde{1}) \subset A$.

We will denote the set of all IVQIs of $S$ as IVQI(S).

Example 4.1. Let $S=\{a, b, c\}$ be any semigroup with the following multiplication table:

We define a mapping $A: S \rightarrow D(I)$ as follows:

$A(a)=[0.1,0.8], A(b)=[0.1,0.8], A(c)=[0.3,0.6]$.

Then we can see that $A \in \operatorname{IVQI}(\mathrm{S})$.

Theorem 4.2. Let $A$ be a nonempty subset of a semigroup $S$. Then $A \in \mathrm{QI}(\mathrm{S})$ if and only if $\left[\chi_{A}, \chi_{A}\right] \in \mathrm{IVQI}(\mathrm{S})$. 


\begin{tabular}{c|ccc} 
& $a$ & $b$ & $c$ \\
\hline$a$ & $a$ & $a$ & $a$ \\
$b$ & $a$ & $b$ & $b$ \\
$c$ & $a$ & $a$ & $b$
\end{tabular}

Proof. ( $\Rightarrow$ ): Suppose $A \in \mathrm{QI}(\mathrm{S})$ and let $x \in S$. Suppose $x \in A$. Then clearly

$$
\chi_{A}(x)=1 \geq\left(\left(\tilde{1} \circ\left[\chi_{A}, \chi_{A}\right]\right) \cap\left(\left[\chi_{A}, \chi_{A}\right] \circ \tilde{1}\right)\right)^{L}(x) .
$$

Thus $\left(\tilde{1} \circ\left[\chi_{A}, \chi_{A}\right]\right) \cap\left(\left[\chi_{A}, \chi_{A}\right] \circ \tilde{1}\right) \subset\left[\chi_{A}, \chi_{A}\right]$. Suppose $x \notin A$. Then either $x$ is expressible as $x=y z$ or not.

Case (i): Suppose $x$ is not expressible as $x=y z$. Then

$\left(\left(\tilde{1} \circ\left[\chi_{A}, \chi_{A}\right]\right) \cap\left(\left[\chi_{A}, \chi_{A}\right] \circ \tilde{1}\right)\right)(x)=\tilde{0}=\left[\chi_{A}, \chi_{A}\right](x)$.

Case (ii): Suppose $x$ is expressible as $x=y z$. Since $x \notin A$, either $y \in A$ or $z \notin A$. If $y \in A$ and $z \notin A$, then there cannot be another expression of the form $x=a b$, where $a \notin A$ and $b \in A$ (Assume that there exist $a \notin A$ and $b \in A$ such that $x=a b$. Then $x \in S A \cap A S \subset$ $A$. Thus $x \in A$. This contradicts the fact that $x \notin A)$. Thus either $\left(\tilde{1} \circ\left[\chi_{A}, \chi_{A}\right]\right)(x)=\tilde{0}$ or $\left(\left[\chi_{A}, \chi_{A}\right] \circ \tilde{1}\right)(x)=$ $\tilde{0}$. So $\left.\left(\tilde{1} \circ\left[\chi_{A}, \chi_{A}\right]\right) \cap\left(\left[\chi_{A}, \chi_{A}\right] \circ \tilde{1}\right)\right)(x)=\tilde{0}$. Then $\left(\tilde{1} \circ\left[\chi_{A}, \chi_{A}\right]\right) \cap\left(\left[\chi_{A}, \chi_{A}\right] \circ \tilde{1}\right) \subset\left[\chi_{A}, \chi_{A}\right]$. Hence, in all, $\left[\chi_{A}, \chi_{A}\right] \in \operatorname{IVQI}(\mathrm{S})$.

$(\Leftarrow)$ : Suppose the necessary condition holds. Let $x \in$ $S A \cap A S$. Then $x \in S A$ and $x \in A S$. Thus there exist $a, a^{\prime} \in A$ and $s, s^{\prime} \in S$ such that $x=s a$ and $x=a^{\prime} s^{\prime}$. So

$$
\begin{aligned}
((\tilde{1} \circ & {\left.\left.\left[\chi_{A}, \chi_{A}\right]\right) \cap\left(\left[\chi_{A}, \chi_{A}\right] \circ \tilde{1}\right)\right)^{L}(x) } \\
& =\left(\tilde{1} \circ\left[\chi_{A}, \chi_{A}\right]\right)^{L}(x) \wedge\left(\left[\chi_{A}, \chi_{A}\right] \circ \tilde{1}\right)^{L}(x) \\
& =\bigvee_{x=y z}\left(\tilde{1}^{L}(y) \wedge \chi_{A}(z)\right) \wedge \bigvee_{x=p q}\left(\chi_{A}(p) \wedge \chi_{S}(q)\right) \\
& \geq\left(\chi_{S}(s) \wedge \chi_{A}(a)\right) \wedge\left(\chi_{A}\left(a^{\prime}\right) \wedge \tilde{1}^{L}\left(s^{\prime}\right)\right) \\
& \quad\left(\text { Since } x=s a \text { and } x=a^{\prime} s^{\prime}\right) \\
& =1 .
\end{aligned}
$$

Similarly, we have that $\left(\left(\tilde{1} \circ\left[\chi_{A}, \chi_{A}\right]\right) \cap\left(\left[\chi_{A}, \chi_{A}\right] \circ\right.\right.$ 1) $)^{U}(x) \geq 1$. Then, by the hypothesis, $\chi_{A}(x) \geq 1$. Thus $x \in A$. So $S A \cap A S \subset A$. Hence $A \in \mathrm{QI}(\mathrm{S})$. This completes the proof.

Definition 4.3. [1] A nonempty fuzzy set $A$ of a semigroup $S$ is called a fuzzy quasi-ideal of $S$ if $\left(\chi_{S} \circ A\right) \wedge\left(A \circ \chi_{S}\right) \leq$ $A$, where $\chi_{S}$ is the whole fuzzy set defined by $\chi_{S}(x)=1$ for each $x \in S$.

Remark 4.3 Let $S$ be a semigroup.

(a) If $A$ is a fuzzy quasi-ideal of $S$, then $[A, A] \in \mathrm{IVQI}(\mathrm{S})$.

(b) If $A \in \operatorname{IVQI}(\mathrm{S})$, then $A^{L}$ and $A^{U}$ are fuzzy quasi-ideals of $S$.

Proposition 4.4. Let $S$ be a semigroup. Then IVQI(S) $\subset$ IVSG(S).
Proof. Let $A \in \mathrm{IVQI}(\mathrm{S})$. Since $A \subset \tilde{1}$, by Proposition 3.5, $A \circ A \subset \tilde{1} \circ A$ and $A \circ A \subset A \circ \tilde{1}$. Then $A \circ A \subset[\tilde{1} \circ A] \cap$ $[A \circ \tilde{1}]$. Since $A \in \operatorname{IVQI}(\mathrm{S}),(\tilde{1} \circ A) \cap(A \circ \tilde{1}) \subset A$. Thus $A \circ A \subset A$. Hence, by Proposition 3.4, $A \in \mathrm{IVSG}(\mathrm{S})$.

Proposition 4.5. Let $S$ be a semigroup. Then IVLI(S) $\subset$ $\operatorname{IVQI}(\mathrm{S})$ and IVRI $(\mathrm{S}) \subset \mathrm{IVQI}(\mathrm{S})$.

Proof. Let $A \in \operatorname{IVLI}(\mathrm{S})$. Then, by Result 3.C, $\tilde{1} \circ A \subset A$. Thus $(\tilde{1} \circ A) \cap[A \circ \tilde{1}] \subset \tilde{1} \circ A \subset A$. Hence $A \in \operatorname{IVQI}(\mathrm{S})$. Similarly, we can see that IVRI(S) $\subset$ IVQI(S).

Proposition 4.6. Let $S$ be a semigroup, let $A \in \operatorname{IVLI}(\mathrm{S})$ and let $B \in \operatorname{IVRI}(\mathrm{S})$. Then $A \cap B \in \mathrm{IVQI}(\mathrm{S})$.

Proof. Let $A \in \operatorname{IVLI}(\mathrm{S})$ and let $B \in \operatorname{IVRI}(\mathrm{S})$. Then, by Result 3.C, $A \circ \tilde{1} \subset A$ and $\tilde{1} \circ B \subset B$. Thus

$$
\begin{aligned}
&(\tilde{1} \circ(A \cap B)) \cap((A \cap B) \circ \tilde{1}) \\
& \quad \subset \quad((\tilde{1} \circ A) \cap(\tilde{1} \circ B)) \cap((A \circ \tilde{1}) \cap(B \circ \tilde{1})) \\
& \quad(\text { By Proposition 3.2 (b), 3.5 and Result 3.C }) \\
& \quad \subset \quad((\tilde{1} \circ A) \cap B) \cap(A \circ \cap(B \circ \tilde{1})) \\
& \quad=((\tilde{1} \circ A) \cap(B \circ \tilde{1})) \cap(A \cap B) \\
& \subset \quad A \cap B .
\end{aligned}
$$

Hence $A \cap B \in \operatorname{IVQI}(\mathrm{S})$.

The following is the immediate result of Propositions 3.5 and 4.6.

Corollary 4.6 Let $S$ be a semigroup and let $\tilde{0} \neq A \in$ $D(I)^{S}$. Then $(A \cup(\tilde{1} \circ A)) \cap(A \cup(A \circ \tilde{1})) \in \operatorname{IVQI}(\mathrm{S})$.

Proposition 4.7. Let $S$ be a semigroup and let $A \in$ IVQI(S). Then

$$
A=(A \cup(\tilde{1} \circ A)) \cap(A \cup(A \circ \tilde{1})) .
$$

Proof. It is clear that $(A \cup(\tilde{1} \circ A)) \in \operatorname{IVLI}(\mathrm{S})$ and $(A \cup(A \circ \tilde{1})) \in \operatorname{IVRI}(\mathrm{S})$, from Proposition 3.7. Also from Proposition 4.6, it is clear that $(A \cup(\tilde{1} \circ A)) \cap(A \cup(A \circ \tilde{1})) \in$ IVQI(S). Then it is sufficient to show that the equality holds.

$$
\begin{aligned}
A \subset & (A \cup(\tilde{1} \circ A)) \cap(A \cup(A \circ \tilde{1})) \\
& (\text { Since } A \subset A \cup(\tilde{1} \circ A) \text { and } A \subset A \cup(A \circ \tilde{1})) \\
= & ((A \cup(\tilde{1} \circ A)) \cap A) \cup((A \cup(\tilde{1} \circ A)) \cap(A \circ \tilde{1}))
\end{aligned}
$$

(By Proposition 3.2)

$$
\begin{gathered}
\subset A \cup((A \cup(\tilde{1} \circ A)) \cap(A \circ \tilde{1})) \\
\quad(\text { Since }(A \cup(\tilde{1} \circ A)) \cap A \subset A) \\
=A \cup(A \cap(A \circ \tilde{1})) \cup((\tilde{1} \circ A) \cap(A \circ \tilde{1}))
\end{gathered}
$$

(By Proposition 3.2)

$$
\begin{aligned}
& \subset A \cup(A \cap(A \circ \tilde{1})) \cup A \\
& \quad(\operatorname{Since}(\tilde{1} \circ A) \cap(A \circ \tilde{1}) \subset A)
\end{aligned}
$$




$$
\begin{aligned}
& \subset A \cup A \cup A \\
& \quad(\text { Since } A \cap(A \circ \tilde{1}) \subset A) \\
& =A .
\end{aligned}
$$

Hence, the equality holds.

The following is the immediate result of Propositions 4.6 and 4.7 .

Theorem 4.8. Let $S$ be a semigroup and let $\tilde{0} \neq A \in$ $D(I)^{S}$. Then $A \in \mathrm{IVQI}(\mathrm{S})$ if and only if there exist $B \in$ IVRI(S) and $C \in \operatorname{IVLI}(\mathrm{S})$ such that $A=B \cap C$.

Proposition 4.9. Let $S$ be a semigroup and let $\left\{A_{\alpha}\right\}_{\alpha \in \Gamma} \subset$ IVQI(S). Then either $\bigcap_{\alpha \in \Gamma} A_{\alpha}=\tilde{0}$ or $\bigcap_{\alpha \in \Gamma} A_{\alpha} \in \operatorname{IVQI}(\mathrm{S})$.

Proof. Let $\left\{A_{\alpha}\right\}_{\alpha \in \Gamma} \subset \mathrm{IVQI}(\mathrm{S})$ and let $A=\bigcap_{\alpha \in \Gamma} A_{\alpha}$. Suppose $A \neq \tilde{0}$. Then

$$
\begin{aligned}
(\tilde{1} \circ A) \cap & (A \circ \tilde{1})=\left(\tilde{1} \circ \bigcap_{\alpha \in \Gamma} A_{\alpha}\right) \cap\left(\bigcap_{\alpha \in \Gamma} A_{\alpha} \circ \tilde{1}\right) \\
& \subset \bigcap_{\alpha \in \Gamma}\left(\tilde{1} \circ A_{\alpha}\right) \cap\left(\bigcap_{\alpha \in \Gamma}\left(A_{\alpha} \circ \tilde{1}\right)\right) \\
& \subset \bigcap_{\alpha \in \Gamma}\left(\left(\tilde{1} \circ A_{\alpha}\right) \cap\left(A_{\alpha} \circ \tilde{1}\right)\right) \\
& \subset \bigcap_{\alpha \in \Gamma} A_{\alpha} \\
& =A .
\end{aligned}
$$

Hence $A=\bigcap_{\alpha \in \Gamma} A_{\alpha} \in \mathrm{IVQI}(\mathrm{S})$.

A nonempty subset $A$ of a semigroup $S$ is called a $b i$ ideal [7] of $S$ if $A^{2} \subset A$ and $A S A \subset A$. We will denote the set of all bi-ideals of $S$ as $\operatorname{BI}(\mathrm{S})$.

Definition 4.10. [8]. Let $S$ be a semigroup and let $\tilde{0} \neq$ $A \in D(I)^{S}$. Then $A$ is called an interval-valued fuzzy bi-ideal (in short, $I V B I$ ) of $S$ if it satisfies the following conditions: for any $x, y, z \in S$.

(a) $A^{L}(x y) \geq A^{L}(x) \wedge A^{L}(y)$ and $A^{U}(x y) \geq A^{U}(x) \wedge$ $A^{U}(y)$

(b) $A^{L}(x y z) \geq A^{L}(x) \wedge A^{L}(z)$ and $A^{U}(x y z) \geq$ $A^{U}(x) \wedge A^{U}(z)$.

We will denote the set of all IVBIs of $S$ as IVBI(S).

Result 4.A. $[8$, Theorem 2.8]. Let $A$ be a nonempty subset of a semigroup. Then $A \in \mathrm{BI}(\mathrm{S})$ if and only if $\left[\chi_{A}, \chi_{A}\right] \in$ $\operatorname{IVBI}(\mathrm{S})$.

Theorem 4.11. Let $S$ be a semigroup and let $\tilde{0} \neq A \in$ $D(I)^{S}$. Then $A \in \operatorname{IVBI}(\mathrm{S})$ if and only if $A \circ A \subset A$ and $A \circ \tilde{1} \circ A \subset A$.
Proof. $(\Rightarrow)$ : Suppose $A \in \operatorname{IVBI}(\mathrm{S})$. From Proposition 3.4, $A \circ A \subset A$. Let $x \in S$. Suppose $x$ is not expressible as $x=y z$. Then clearly $(A \circ \tilde{1} \circ A)(x)=\tilde{0}$. Thus $A \circ$ $\tilde{1} \circ A \subset A$. Suppose $x$ is expressible as $x=y z$. Then $(A \circ \tilde{1} \circ A)(x) \neq \tilde{0}$. Thus

$$
(A \circ \tilde{1} \circ A)^{L}(x)=\bigvee_{x=y z}\left(A^{L}(y) \wedge(\tilde{1} \circ A)^{L}(z)\right)>0
$$

and

$$
(A \circ \tilde{1} \circ A)^{U}(x)=\bigvee_{x=y z}\left(A^{U}(y) \wedge(\tilde{1} \circ A)^{U}(z)\right)>0 .
$$

So $(\tilde{1} \circ A)^{L}(z)>0$ and $(\tilde{1} \circ A)^{U}(z)>0$. Then there exist $p, q \in S$ with $z=p q$ such that

$$
(\tilde{1} \circ A)^{L}(z)=\bigvee_{z=p q}\left(\tilde{1}^{L}(p) \wedge A^{L}(q)\right)=\bigvee_{z=p q} A^{L}(q)
$$

and

$$
(\tilde{1} \circ A)^{U}(z)=\bigvee_{z=p q}\left(\tilde{1}^{U}(p) \wedge A^{U}(q)\right)=\bigvee_{z=p q} A^{U}(q) .
$$

Since $A \in \operatorname{IVBI}(\mathrm{S})$,

$$
A^{L}(x)=A^{L}(y p q) \geq A^{L}(y) \wedge A^{L}(q)
$$

and

$$
A^{U}(x)=A^{U}(y p q) \geq A^{U}(y) \wedge A^{U}(q)
$$

Then

$A^{L}(x) \geq \bigvee_{x=y z}\left(A^{L}(y) \wedge\left(\bigvee_{z=p q} A^{L}(q)\right)\right)=(A \circ \tilde{1} \circ A)^{L}(x)$

and

$A^{U}(x) \leq \bigvee_{x=y z}\left(A^{U}(y) \wedge\left(\bigvee_{z=p q} A^{U}(q)\right)\right)=(A \circ \tilde{1} \circ A)^{U}(x)$

Hence, in all, $A \circ \tilde{1} \circ A \subset A$.

$(\Leftarrow)$ : Suppose the necessary condition holds. Since $A \circ A \subset A$, it is clear that the following hold: $A^{L}(x y) \geq A^{L}(x) \wedge A^{L}(y)$ and $A^{U}(x y) \geq A^{U}(x) \wedge$ $A^{U}(y)$ for any $x, y \in S$. Let $x, y, z \in S$ and let $p=x y z$. Then

$$
\begin{aligned}
A^{L}(x y z)= & A^{L}(p) \geq(A \circ \tilde{1} \circ A)^{L}(p) \\
& (\text { By the hypothesis }) \\
= & \bigvee_{p=s t}\left(A^{L}(s) \wedge(\tilde{1} \circ A)^{L}(t)\right) \\
\geq & A^{L}(x) \wedge(\tilde{1} \circ A)^{L}(y z) \quad(\text { Since } p=x(y z)) \\
= & A^{L}(x) \wedge\left(\bigvee_{y z=a b}\left(\tilde{1}^{L}(a) \wedge A^{L}(b)\right)\right) \\
\geq & A^{L}(x) \wedge \tilde{1}^{L}(y) \wedge A^{L}(z) \\
= & A^{L}(x) \wedge A^{L}(z) .
\end{aligned}
$$

Similarly, we have that $A^{U}(x y z) \geq A^{U}(x) \wedge A^{U}(z)$. Hence, $A \in \operatorname{IVBI}(\mathrm{S})$. This completes the proof. 
Proposition 4.12. Let $S$ be a semigroup. Then IVQI(S) $\subset$ $\operatorname{IVBI}(\mathrm{S})$.

Proof. Let $A \in \mathrm{IVQI}(\mathrm{S})$. Then, by Proposition 4.4, $A \in$ IVSG(S). Thus $A^{L}(x y) \geq A^{L}(x) \wedge A^{L}(y)$ and $A^{U}(x y) \geq$ $A^{U}(x) \wedge A^{U}(y)$ for any $x, y \in S$. So, by Proposition 3.4, $A \circ A \subset A$. It is clear that $A \circ \tilde{1} \subset \tilde{1}$ and $\tilde{1} \circ A \subset \tilde{1}$. Then, by Proposition 3.5, $A \circ \tilde{1} \circ A \subset \tilde{1} \circ A$ and $A \circ \tilde{1} \circ A \subset A \circ \tilde{1}$. Thus $A \circ \tilde{1} \circ A \subset[\tilde{1} \circ A] \cap[A \circ \tilde{1}] \subset A$ (Since $A \in \operatorname{IVQI}(\mathrm{S})$ ). Hence, by Theorem 4.11, $A \in \operatorname{IVBI}(\mathrm{S})$.

The converse inclusion of Proposition 4.12 is not generally true.

Example 4.2. Let $S=\{a, b, c, d\}$ be the semigroup with the following multiplication table:

\begin{tabular}{c|cccc} 
& $a$ & $b$ & $c$ & $d$ \\
\hline$a$ & $a$ & $a$ & $a$ & $a$ \\
$b$ & $a$ & $a$ & $a$ & $a$ \\
$c$ & $a$ & $a$ & $a$ & $b$ \\
$d$ & $a$ & $a$ & $b$ & $c$
\end{tabular}

We define a mapping $A: S \rightarrow D(I)$ as follows:

$$
A(a)=[1,1], A(b)=[0.3,0.6], A(c)=[0.2,0.7]
$$

and

$$
A(d)=[0.5,0.5] .
$$

Then we can see that $A \notin \mathrm{IVQI}(\mathrm{S})$ but $A \in \operatorname{IVBI}(\mathrm{S})$.

The product of two quasi-ideals need not be a quasiideal. So the interval-valued fuzzy product of two IVQIs need not be an IVQI.

Proposition 4.13. Let $S$ be a semigroup, let $A \in \operatorname{IVQI}(\mathrm{S})$ and let $\tilde{0} \neq B \in D(I)^{S}$. Then $A \circ B, B \circ A \in \operatorname{IVBI}(\mathrm{S})$.

Proof. Let $A \in \mathrm{IVQI}(\mathrm{S})$ and let $\tilde{0} \neq B \in D(I)^{S}$. Thus, by Proposition 4.12, $A \in \operatorname{IVBI}(\mathrm{S})$. Then, by Theorem 4.11, $A \circ \tilde{1} \circ A \subset A$ and $A \circ A \subset A$. So

$$
\begin{aligned}
(A \circ B) \circ & (A \circ B) \subset(A \circ \tilde{1}) \circ(A \circ B) \\
& (\text { Since } A \circ B \subset A \circ \tilde{1}) \\
= & (A \circ \tilde{1} \circ A) \circ B \\
& (\text { By Result 4.A }) \\
\subset A \circ B . &
\end{aligned}
$$

(Since $A \circ \tilde{1} \circ A \subset A$ )

On the other hand,

$$
\begin{aligned}
(A \circ B) \circ \tilde{1} \circ( & (A \circ B) \\
& \subset(A \circ \tilde{1}) \circ \tilde{1} \circ(A \circ B) \\
& \quad(\text { Since } A \circ B \subset A \circ \tilde{1})
\end{aligned}
$$

$$
\begin{aligned}
= & A \circ(\tilde{1} \circ \tilde{1}) \circ(A \circ B) \\
& \quad(\text { By Result 3.A }) \\
\subset & A \circ \tilde{1} \circ(A \circ B) \\
= & (A \circ \tilde{1} \circ A) \circ B
\end{aligned}
$$

(By Result 3.A)

$\subset A \circ B . \quad($ Since $A \circ \tilde{1} \circ A \subset A)$

Hence, by Theorem 4.11, $A \circ B \in \operatorname{IVBI}(\mathrm{S})$. Similarly, we can see that $B \circ A \in \operatorname{IVBI}(\mathrm{S})$. This completes the proof.

The following is the immediate result of Proposition 4.13 .

Corollary 4.13 Let $S$ be a semigroup and let $A, B \in$ IVQI(S). Then $A \circ B \in \operatorname{IVBI}(\mathrm{S})$.

\section{Regular semigroups}

A semigroup $S$ is said to be regular if for each $a \in S$ there exists $x \in S$ such that $a=a x a$.

Theorem 5.1. Let $S$ be a semigroup. Then the following are equivalent :

(a) $S$ is regular.

(b) For each $A \in \operatorname{IVRI}(\mathrm{S})$ and each $B \in \operatorname{IVLI}(\mathrm{S}), A \circ$ $B=A \cap B$.

(c) For each $A \in \operatorname{IVRI}(\mathrm{S})$ and each $B \in \operatorname{IVLI}(\mathrm{S})$,
(1) $A^{2}=A \circ A=A$.
(2) $B^{2}=B \circ B=B$.
(3) $A \circ B \in \mathrm{IVQI}(\mathrm{S})$.

(d) $(\mathrm{IVQI}(\mathrm{S}), \circ)$ is a regular semigroup.

(e) Every IVQI $A$ of $S$ has the form $A=A \circ \tilde{1} \circ A$.

Proof. (a) $\Rightarrow$ (b) : Suppose $S$ is regular. Let $A \in \operatorname{IVRI}(\mathrm{S})$ and let $B \in \operatorname{IVLI}(\mathrm{S})$. Then, by Result 3.C, $A \circ B \subset A \circ \tilde{1} \subset$ $A$ and $A \circ B \subset \tilde{1} \circ B \subset B$. Thus $A \circ B \subset A \cap B$.

Now let $a \in S$. Since $S$ is regular, there exists an $x \in S$ such that $a=a x a$. Then

$$
\begin{aligned}
(A \circ B)^{L}(a)= & \bigvee_{a=y z}\left[A^{L}(y) \wedge B^{L}(z)\right] \geq A^{L}(a x) \wedge B^{L}(a) \\
& \quad(\text { Since } a=a x a) \\
\geq & \left.A^{L}(a) \wedge B^{L}(a) \quad \text { Since } A \in \operatorname{IVRI}(\mathrm{S})\right) \\
= & (A \cap B)^{L}(a)
\end{aligned}
$$

Similarly, we have that

$$
(A \circ B)^{U}(a) \geq(A \cap B)^{U}(a)
$$

Thus $A \circ B \supset A \cap B$. Hence $A \circ B=A \cap B$. (b) $\Rightarrow$ (c) : Suppose the condition (b) holds. 
(1) Let $A \in \operatorname{IVRI}(\mathrm{S})$. Then, by Proposition 3.8, $A \cup(\tilde{1} \circ$ $A) \in \operatorname{IVI}(\mathrm{S})$. By the hypothesis,

$$
\begin{aligned}
A= & A \cap(A \cup(\tilde{1} \circ A))=A \circ(A \cup(\tilde{1} \circ A)) \\
= & (A \circ A) \cup(A \circ(\tilde{1} \circ A)) \\
& \quad(\text { By Proposition 3.2 (a) }) \\
= & (A \circ A) \cup((A \circ \tilde{1}) \circ A) \quad(\text { By Result 3.A }) \\
\subset & (A \circ A) \cup(A \circ A) \quad(\text { Since } A \in \operatorname{IVRI}(\mathrm{S})) \\
= & (A \circ A) .
\end{aligned}
$$

So $A \subset A \circ A$. On the other hand, $A \circ A \subset A \circ \tilde{1} \subset A$. Hence $A \circ A=A$.

(2) Let $B \in \operatorname{IVLI}(\mathrm{S})$. Then, by the similar arguments of the proof of (1), we can see that $B \circ B=B$.

(3) Let $A \in \operatorname{IVRI}(\mathrm{S})$ and let $B \in \operatorname{IVLI}(\mathrm{S})$. Then, by the hypothesis, $A \circ B=A \cap B$. By Proposition 4.6, $A \cap B \in$ IVQI(S). Hence $A \circ B \in \operatorname{IVQI(S).~}$

(c) $\Rightarrow$ (d): Suppose the condition (c) holds. Let $A \in$ IVQI(S). Then, by Proposition 3.7, $A \cup(\tilde{1} \circ A) \in \operatorname{IVLI}(\mathrm{S})$. Thus

$$
\begin{aligned}
A \subset A \cup(\tilde{1} \circ A) & (A \cup(\tilde{1} \circ A)) \circ(A \cup(\tilde{1} \circ A)) \\
& \quad(\text { By the condition }(\mathrm{c})(2)) \\
= & ((A \cup(\tilde{1} \circ A) \circ A) \cup((A \cup(\tilde{1} \circ A)) \circ(\tilde{1} \circ A)) \\
& \quad(\text { By Proposition 3.2 }(\mathrm{a})) \\
= & ((A \circ A) \cup\{(\tilde{1} \circ A) \circ A\}) \cup\left((A \circ(\tilde{1} \circ A)) \cup(\tilde{1} \circ A)^{2}\right. \\
& \quad(\text { By Proposition 3.2(a)) } \\
= & ((A \circ A) \cup\{\tilde{1} \circ(A \circ A)\}) \cup\left((A \circ(\tilde{1} \circ A)) \cup(\tilde{1} \circ A)^{2}\right) \\
& \quad(\text { By Result } 3 . \mathrm{A}) \\
\subset & ((\tilde{1} \circ A) \cup(\tilde{1} \circ A)) \cup\left((\tilde{1} \circ(\tilde{1} \circ A)) \cup(\tilde{1} \circ A)^{2}\right. \\
= & ((\tilde{1} \circ A) \cup(\tilde{1} \circ A)) \cup((\tilde{1} \circ(\tilde{1} \circ A)) \cup(\tilde{1} \circ A) \\
\quad & (\text { By the condition }(\mathrm{c})(2)) \\
\subset & (\tilde{1} \circ A) \cup(\tilde{1} \circ A) \cup(\tilde{1} \circ A) \\
& (\text { Since } \tilde{1} \circ A \in \operatorname{IVLI}(\mathrm{S})) \\
= & \tilde{1} \circ A .
\end{aligned}
$$

So $A \subset \tilde{1} \circ A$. By the similar arguments, we can see that $A \subset A \circ \tilde{1}$. Then $A \subset(\tilde{1} \circ A) \cap(A \circ \tilde{1})$. Since $A \in \operatorname{IVQI}(\mathrm{S})$, $(\tilde{1} \circ A) \cap(A \circ \tilde{1}) \subset A$. So

$$
A=(\tilde{1} \circ A) \cap(A \circ \tilde{1}) .
$$

Let $C \in \operatorname{IVRI}(\mathrm{S})$ and let $D \in \operatorname{IVLI}(\mathrm{S})$. Then, by the condition (c)(3), $C \circ D \in \operatorname{IVQI}(\mathrm{S})$. Thus, by (5.1),

$$
C \circ D=(\tilde{1} \circ(C \circ D)) \cap((C \circ D) \circ \tilde{1}) .
$$

Now let $A, B \in \operatorname{IVQI}(\mathrm{S})$. Then, by Proposition 3.6, $\tilde{1} \circ A \circ B \in \operatorname{IVLI}(\mathrm{S})$ and $A \circ B \circ \tilde{1} \in \operatorname{IVRI}(\mathrm{S})$. By Propo- sition 4.5, $\tilde{1} \circ A \circ B, A \circ B \circ \tilde{1} \in \mathrm{IVQI}(\mathrm{S})$. Thus

$$
\begin{aligned}
& \tilde{1} \circ A \circ B=(\tilde{1} \circ(\tilde{1} \circ A \circ B)) \cap((\tilde{1} \circ A \circ B) \circ \tilde{1}) \\
&(B y(5.1)) \\
&=(\tilde{1} \circ A \circ B) \circ(\tilde{1} \circ A \circ B) \\
&(\text { By }(5.2)) \\
&=((\tilde{1} \circ A \circ B) \circ \tilde{1}) \circ(\tilde{1} \circ(\tilde{1} \circ A \circ B)) \\
&=(\tilde{1} \circ A \circ B) \circ(\tilde{1} \circ \tilde{1}) \circ(\tilde{1} \circ A \circ B) \\
&=(\tilde{1} \circ A \circ B) \circ \tilde{1} \circ(\tilde{1} \circ A \circ B) .
\end{aligned}
$$

(By the condition (c)(2))

So $\tilde{1} \circ A \circ B=(\tilde{1} \circ A \circ B) \circ \tilde{1} \circ(\tilde{1} \circ A \circ B)$. Similarly, we have that

$$
A \circ B \circ \tilde{1}=(A \circ B \circ \tilde{1}) \circ \tilde{1} \circ(A \circ B \circ \tilde{1}) .
$$

Then

$$
\begin{aligned}
(\tilde{1} \circ & A \circ B) \cap(A \circ B \circ \tilde{1}) \\
= & ((\tilde{1} \circ A \circ B) \circ \tilde{1} \circ(\tilde{1} \circ A \circ B)) \cap((A \circ B \circ \tilde{1}) \\
& \circ \tilde{1} \circ(A \circ B \circ \tilde{1})) \\
= & (\tilde{1} \circ(A \circ B \circ \tilde{1}) \circ(\tilde{1} \circ A \circ B)) \cap((A \circ B \circ \tilde{1}) \\
& \circ(\tilde{1} \circ A \circ B) \circ \tilde{1})) \\
= & (A \circ B \circ \tilde{1}) \circ(\tilde{1} \circ A \circ B) \subset(A \circ B \circ \tilde{1}) \circ \tilde{1} \circ(\tilde{1} \circ B) \\
& \quad(B y(5.2) \text { and } A \subset \tilde{1}) \\
= & (A \circ B \circ \tilde{1}) \circ(\tilde{1} \circ \tilde{1}) \circ B \\
= & (A \circ B \circ \tilde{1}) \circ \tilde{1} \circ B \\
& \quad(\operatorname{Since} \tilde{1} \circ \tilde{1}=\tilde{1}) \\
= & (A \circ B) \circ(\tilde{1} \circ \tilde{1}) \circ B \\
= & A \circ(B \circ \tilde{1} \circ B) \\
& \subset A \circ B .
\end{aligned}
$$

( Since $B \circ \tilde{1} \circ B \subset B$ )

So $A \circ B \in \operatorname{IVQI}(\mathrm{S})$. Since " $\circ$ " is associative , (IVQI(S), $\circ$ ) is a semigroup. Let $A \in \operatorname{IVQI}(\mathrm{S})$. Then

$$
\begin{array}{rlr}
A & =(A \circ \tilde{1}) \cap(\tilde{1} \circ A) & (\text { By }(5.1)) \\
& =(A \circ \tilde{1}) \circ(\tilde{1} \circ A) & (\text { By the condition (3) }) \\
& =A \circ \tilde{1} \circ A .
\end{array}
$$

It is clear that $\tilde{1} \in \operatorname{IVQI}(\mathrm{S})$. So $A$ is a regular element of IVQI(S). Hence (IVQI(S), o) is a regular semigroup.

(d) $\Rightarrow($ e): Suppose the condition (d) holds. Let $A \in$ IVQI(S). Then, by the hypothesis, there exists $B \in$ IVQI(S) such that $A=A B A$. Thus

$$
\begin{aligned}
A= & A B A=A \circ \tilde{1} \circ A \\
& \quad(\text { Since } B \subset \tilde{1}) \\
\subset & (A \circ \tilde{1}) \cap(\tilde{1} \circ A) \\
\subset & A .
\end{aligned}
$$


Hence $A=A \circ \tilde{1} \circ A$.

(e) $\Rightarrow$ (a) : Suppose the condition (e) holds. Let $x \in S$ and let $A=\{x\} \cup(S x \cap x S)$ be the quasi-ideal of $S$ generated by $x$. Then, by Theorem 4.2, $\left[\chi_{A}, \chi_{A}\right] \in \mathrm{IVQI}(\mathrm{S})$. Thus, by the hypothesis, $\left[\chi_{A}, \chi_{A}\right]=\tilde{1} \circ \tilde{1} \circ\left[\chi_{A}, \chi_{A}\right]$. So

$$
\begin{aligned}
1 & =\chi_{A}(x)=\left(\left[\chi_{A}, \chi_{A}\right] \circ \tilde{1} \circ\left[\chi_{A}, \chi_{A}\right]\right)^{L}(x) \\
& =\bigvee_{x=y z}\left(\left(A^{L}(y) \wedge\left(\tilde{1} \circ\left[\chi_{A}, \chi_{A}\right]\right)^{L}(z)\right) .\right.
\end{aligned}
$$

Then there exist $p, q \in S$ with $x=p q$ such that

$$
\left.\chi_{A}(p)=1 \quad \text { and } \quad\left(\tilde{1} \circ\left[\chi_{A}, \chi_{A}\right)\right]\right)^{L}(q)=1 .
$$

Since $\chi_{A}(p)=1, p \in A$, i.e., $p=x$ or $p=x s$ where $s \in S$. Since $\left(\tilde{1} \circ\left[\chi_{A}, \chi_{A}\right]\right)(q)=[1,1]$,

$$
\bigvee_{q=s t}\left(\tilde{1}^{L}(s) \wedge \chi_{A}(t)\right)=1 \text { and } \bigvee_{q=s t}\left(\tilde{1}^{L}(s) \wedge \chi_{A}(t)\right)=1
$$

Then there exist $a, b \in S$ with $q=a b$ such that $\chi_{A}(b)=1$. So $b \in A$, i.e., either $b=x$ or $b=s_{1} x$ where $s_{1} \in S$. Hence $x=p q=x c x$ where $c \in S$. Therefore $x$ is a regular element of $S$. Hence $S$ is regular. This completes the proof.

Theorem 5.2. Let $S$ be a regular semigroup and let $\tilde{0} \neq$ $A \in D(I)^{S}$. Then $A \in \mathrm{IVQI}(\mathrm{S})$ if and only if there exist $B \in \operatorname{IVRI}(\mathrm{S})$ and $C \in \operatorname{IVLI}(\mathrm{S})$ such that $A=B \circ C$.

Proof. $(\Rightarrow)$ : Suppose $A \in \operatorname{IVQI}(\mathrm{S})$. Then

$$
\begin{aligned}
A & =A \circ \tilde{1} \circ A \quad(\text { By Theorem } 5.1) \\
& =A \circ(\tilde{1} \circ \tilde{1}) \circ A \\
& =(A \circ \tilde{1}) \circ(\tilde{1} \circ A) .
\end{aligned}
$$

Let $A \circ \tilde{1}=B$ and let $C=\tilde{1} \circ A$. Then, by Proposition 3.6, $B \in \operatorname{IVRI}(\mathrm{S})$ and $C \in \operatorname{IVLI}(\mathrm{S})$. Hence the necessary condition holds.

$(\Leftarrow)$ : Suppose the necessary condition holds. Let $A \in$ $D(I)^{S}$. Then there exist $B \in \operatorname{IVRI}(\mathrm{S})$ and $C \in \operatorname{IVLI}(\mathrm{S})$ such that $A=B \circ C$. Since $S$ is regular, by Theorem 5.1, $B \circ C \in \operatorname{IVQI}(\mathrm{S})$. Hence $A \in \mathrm{IVQI}(\mathrm{S})$. This completes the proof.

Theorem 5.3. Let $S$ be a regular semigroup. Then the following hold:

(a) If $A \in \operatorname{IVQI}(\mathrm{S})$, then $A^{2}=A^{3}$.

(b) $A \in \operatorname{IVQI}(\mathrm{S})$ if and only if $A \in \operatorname{IVBI}(\mathrm{S})$.

Proof. (a) Suppose $A \in \operatorname{IVQI}(\mathrm{S})$. Since $A \in \operatorname{IVSG}(\mathrm{S}), A \circ$ $A \subset A$. Thus $A \circ(A \circ A) \subset A \circ A$. So $A^{3} \subset A^{2}$. Since $S$ is regular, by Theorem 5.1, $A \circ A \in \operatorname{IVQI}(\mathrm{S})$. Since IVQI(S) is regular, there exists $B \in \mathrm{IVQI}(\mathrm{S})$ such that $A^{2}=A^{2} \circ$ $B \circ A^{2}$. On the other hand, $A^{2} \circ B \circ A^{2} \subset A^{2} \circ \tilde{1} \circ A^{2}=$ $A \circ(A \circ \tilde{1} \circ A) \circ A=A \circ A \circ A=A^{3}$. Thus $A^{2} \subset A^{3}$. Hence $A^{2}=A^{3}$. (b) $(\Rightarrow)$ : It is clear from Proposition 4.12.

$(\Leftarrow)$ : Suppose $A \in \operatorname{IVBI}(\mathrm{S})$. Then $\tilde{1} \circ A \in \operatorname{IVLI}(\mathrm{S})$ and $A \circ \tilde{1} \in \operatorname{IVRI}(\mathrm{S})$. Thus

$$
\begin{aligned}
& {[A \circ \tilde{1}] \cap[\tilde{1} \circ A]=[A \circ \tilde{1}] \circ[\tilde{1} \circ A]} \\
& \text { (By Theorem 5.1) } \\
& =A \circ(\tilde{1} \circ \tilde{1}) \circ A \\
& \text { (By Result 3.A) } \\
& =A \circ \tilde{1} \circ A \subset A \text {. }
\end{aligned}
$$

(By the hypothesis)

Hence $A \in \operatorname{IVQI}(\mathrm{S})$. This completes the proof.

A semigroup $S$ is called a $\operatorname{band}($ See [2]) if for each $a \in S, a a=a$.

Theorem 5.4. Let $S$ be a semigroup. Then the following are equivalent :

(a) For each $A \in \operatorname{IVRI}(\mathrm{S})$ and each $B \in \operatorname{IVLI}(\mathrm{S}), A \circ$ $B=A \cap B \subset B \circ A$.

(b) (IVQI(S), o) is a band.

(c) For each $A \in \operatorname{IVQI}(\mathrm{S}), A \circ A=A$.

Proof. (a) $\Rightarrow$ (b) : Suppose the condition(a) holds. Then, by Theorem 5.1, $S$ is regular. Thus, by Theorem 5.1,(IVQI(S), ०) is a regular semigroup. Let $A \in \mathrm{IVQI}(\mathrm{S})$. Then,

$$
\begin{aligned}
A= & A \circ \tilde{1} \circ A \\
& \quad(\text { By Theorem } 5.1) \\
= & (A \circ \tilde{1} \circ A) \circ \tilde{1} \circ(A \circ \tilde{1} \circ A) \\
& \quad(\text { By theorem } 5.1) \\
= & (A \circ \tilde{1}) \circ(A \circ \tilde{1}) \circ(\tilde{1} \circ A) \circ(\tilde{1} \circ A) \\
& \quad(\text { Since } \tilde{1} \circ \tilde{1}=\tilde{1}) \\
\subset & (A \circ \tilde{1}) \circ(\tilde{1} \circ A) \circ(A \circ \tilde{1}) \circ(\tilde{1} \circ A) \\
& \quad(\text { By the hypothesis }) \\
= & A \circ(\tilde{1} \circ \tilde{1}) \circ A \circ A \circ(\tilde{1} \circ \tilde{1}) \circ A \\
& \quad(\text { By Result } 3 . \mathrm{A}) \\
= & (A \circ \tilde{1} \circ A) \circ(A \circ \tilde{1} \circ A) \\
& \quad(\text { Since } \tilde{1} \circ \tilde{1}=\tilde{1}) \\
= & A \circ A \quad(\text { Since } A \circ \tilde{1} \circ A=A) \\
\subset & A . \quad(\text { Since } A \circ A \subset A)
\end{aligned}
$$

Thus $A \circ A=A$. So $A$ is an idempotent element of IVQI(S). Hence (IVQI(S), o) is a band.

(b) $\Rightarrow$ (c) : It is clear.

(c) $\Rightarrow$ (a) : Suppose the condition (c) holds. Let $A \in$ IVRI(S) and let $B \in \operatorname{IVLI}(\mathrm{S})$. Then, by Proposition 4.6, 
$A \cap B \in \mathrm{IVQI}(\mathrm{S})$. Thus,

$$
\begin{aligned}
A \cap B= & (A \cap B) \circ(A \cap B) \quad(\text { By the hypothesis }) \\
\subset & (A \circ(A \cap B)) \cap(B \circ(A \cap B)) \\
& (\text { By Proposition 3.2(b) }) \\
\subset & (A \circ A) \cap(A \circ B) \cap(B \circ A) \cap(B \circ B) .
\end{aligned}
$$

(By Proposition 3.2(b))

So $A \cap B \subset A \circ B$ and $(A \cap B) \subset B \circ A$.

On the other hand, $A \circ B \subset A \circ \tilde{1} \subset A$ and $A \circ B \subset \tilde{1} \circ B \subset B$. Thus $A \circ B \subset A \cap B$. Hence, by (5.3), $A \circ B=A \cap B \subset B \circ A$. This completes the proof.

A semigroup $S$ is said to be intra-regular [10] if for each $a \in S$ there exist $x, y \in S$ such that $a=x a^{2} y$.

Theorem 5.5. Let $S$ be a semigroup. Then $S$ is intraregular if and only if for each $A \in \operatorname{IVRI(S)}$ and each $B \in$ $\operatorname{IVLI}(\mathrm{S}), A \cap B \subset B \circ A$.

Proof. $(\Rightarrow)$ : Suppose $S$ is intra-regular. Let $A \in \operatorname{IVRI(S),~}$ let $B \in \operatorname{IVLI}(\mathrm{S})$ and let $a \in S$. Then, by the hypothesis, there exist $x, y \in S$ such that $a=x a^{2} y$. Thus

$$
\begin{aligned}
(B \circ A)^{L}(a)= & \bigvee_{a=s t}\left(B^{L}(s) \wedge A^{L}(t)\right) \\
\geq & B^{L}(x a) \wedge A^{L}(a y) \\
& (\text { Since } a=(x a)(a y)) \\
\geq & B^{L}(a) \wedge A^{L}(a)
\end{aligned}
$$

(Since $B \in \operatorname{IVLI}(\mathrm{S})$ and $A \in \operatorname{IVRI}(\mathrm{S})$ )

$$
=(A \cap B)^{L}(a)
$$

Similarly, we have that $(B \circ A)^{U}(a) \geq(A \cap B)^{U}(a)$. Hence $A \cap B \subset B \circ A$.

$(\Leftarrow)$ : Suppose the necessary condition holds and let $x \in S$. Let $L=\{x\} \cup S x$ and $R=\{x\} \cup x S$ be the left and right ideals of $S$ generated by $x$, respectively. Then, by Result 3.B (b), $\left[\chi_{L}, \chi_{L}\right] \in \operatorname{IVLI}(S)$ and $\left[\chi_{R}, \chi_{R}\right] \in \operatorname{IVRI}(S)$. Thus, by the hypothesis,

$$
\left[\chi_{R}, \chi_{R}\right] \cap\left[\chi_{L}, \chi_{L}\right] \subset\left[\chi_{L}, \chi_{L}\right] \circ\left[\chi_{R}, \chi_{R}\right] .
$$

So

$$
\begin{aligned}
\left(\left[\chi_{L}, \chi_{L}\right] \circ\left[\chi_{R}, \chi_{R}\right]\right)^{L} & (x) \\
& =\bigvee_{x=y z}\left(\chi_{L}(y) \wedge \chi_{R}(z)\right) \\
& \geq \chi_{L}(x) \wedge \chi_{R}(x)=1 .
\end{aligned}
$$

Similarly, we have that $\left(\left[\chi_{L}, \chi_{L}\right] \circ\left[\chi_{R}, \chi_{R}\right]\right)^{U}(x) \geq 1$. Then there exist $p, q \in S$ with $x=p q$ such that $\chi_{L}(p)=1$, $\chi_{L}(p)=0$ and $\chi_{R}(q)=1, \chi_{R}(q)=0$. Thus $p \in L$ and $q \in R$. So $p=x$ or $p=s x$ and $q=x$ or $q=x s$ where $s \in S$. In any case, $x=p q=a x^{2} b$, where $a, b \in S$. Hence $S$ is intra-regular. This completes the proof.

The following is the immediate results of Theorems 5.4 and 5.5.

Theorem 5.6. Let $S$ be a semigroup. Then the following are equivalent:

(a) $S$ is regular and intra-regular.

(b) For each $A \in \operatorname{IVRI}(\mathrm{S})$ and each $B \in \operatorname{IVLI}(\mathrm{S}), A \circ$ $B=A \cap B \subset B \circ A$.

(c) (IVQI(S), o) is a band.

(d) For each $A \in \operatorname{IVQI}(\mathrm{S}), A \circ A=A$.

Example 5.7 Let $S=\{a, b, c, d, e\}$ be the semigroup with the following multiplication table:

\begin{tabular}{l|lllll} 
& $a$ & $b$ & $c$ & $d$ & $e$ \\
\hline$a$ & $a$ & $a$ & $a$ & $a$ & $a$ \\
$b$ & $a$ & $a$ & $a$ & $b$ & $c$ \\
$c$ & $a$ & $b$ & $c$ & $a$ & $a$ \\
$d$ & $a$ & $a$ & $a$ & $d$ & $e$ \\
$e$ & $a$ & $d$ & $e$ & $a$ & $a$
\end{tabular}

Then clearly $S$ is a non-commutative semigroup which is not intra-regular. We define two mappings $A, B: S \rightarrow$ $D(I)$ as follows, respectively :

$$
A^{L}(a) \geq A^{L}(x), \quad A^{U}(a) \geq A^{U}(x)
$$

for each $x \in S$,

$$
A(b)=A(d), \quad A(c)=A(e)
$$

and

$$
B^{L}(a) \geq B^{L}(x), \quad B^{U}(a) \geq B^{U}(x)
$$

for each $x \in S$,

$$
B(b)=B(c), \quad B(d)=B(e) .
$$

Then we can easily see that $\tilde{0} \neq A \in \operatorname{IVLI}(\mathrm{S})$ and $\tilde{0} \neq$ $B \in \operatorname{IVRI}(\mathrm{S})$. Moreover, we can check that $A \circ A=A$, $B \circ B=B$ and $B \circ A=B \cap A$. Now we define a mapping $C: S \rightarrow D(I)$ as follows:

$$
C(a)=[1,1], C(b)=C(c)=C(d)=[0.4,0.5]
$$

and

$$
C(e)=[0.5,0.5] .
$$

Then we can see that $A \in \operatorname{IVQI}(\mathrm{S})$ and $A \neq A \circ A=$ $A \circ A \circ A$.

Example 5.8 Let $S=\{0, a, 1\}$ be the semigroup with the following multiplication table: 
International Journal of Fuzzy Logic and Intelligent Systems, vol. 12, no. 3, September 2012

\begin{tabular}{l|lll} 
& 0 & $a$ & 1 \\
\hline 0 & $a$ & $a$ & $a$ \\
$a$ & $a$ & $a$ & $a$ \\
1 & $a$ & $a$ & $b$
\end{tabular}

Then $S$ is a commutative semigroup which is regular and intra-regular. Let $A \in \operatorname{IVQI}(\mathrm{S})$ and let $x \in S$. Then

$$
\begin{aligned}
A^{L}(x) & \geq(A \circ A)^{L}(x) \quad(\text { Since } A \in \operatorname{IVSG}(\mathrm{S})) \\
& =\bigvee_{x=y z}\left(A^{L}(y) \wedge A^{L}(z)\right)=\bigvee_{x=y z} A^{L}(y)
\end{aligned}
$$

(Since $S$ is commutative)

and

$$
\begin{aligned}
A^{U}(x) & \geq(A \circ A)^{U}(x)=\bigvee_{x=y z}\left(A^{U}(y) \wedge A^{U}(z)\right) \\
& =\bigvee_{x=y z} A^{U}(y) .
\end{aligned}
$$

Thus

$$
\begin{aligned}
A^{L}(0) & =\bigvee_{0=y z} A^{L}(y) \geq A^{L}(x), \quad A^{U}(0) \\
& =\bigvee_{0=y z} A^{U}(y) \geq A^{U}(x)
\end{aligned}
$$

for each $x \in S$ and

$$
\begin{aligned}
& A^{L}(a)=A^{L}(a) \wedge A^{L}(1) \geq A^{L}(1), \\
& A^{U}(a)=A^{U}(a) \wedge A^{U}(1) \geq A^{U}(1) .
\end{aligned}
$$

So $A^{L}(0) \geq A^{L}(a) \geq A^{L}(1)$ and $A^{U}(0) \geq A^{U}(a) \geq$ $A^{U}(1)$. Then

$$
\begin{aligned}
(A \circ A)^{L}(0) & =\bigvee_{0=x y}\left(A^{L}(x) \wedge A^{L}(y)\right)=A^{L}(0) \\
(A \circ A)^{U}(0) & =\bigvee_{0=x y}\left(A^{U}(x) \wedge A^{U}(y)\right)^{U}=A^{U}(0), \\
(A \circ A)^{L}(a) & =\left(A^{L}(a) \wedge A^{L}(a)\right) \vee\left(A^{L}(a) \wedge A^{L}(1)\right) \\
& =A^{L}(a), \\
(A \circ A)^{U}(a) & =\left(A^{U}(a) \wedge A^{U}(a)\right) \vee\left(A^{U}(a) \wedge A^{U}(1)\right) \\
& =A^{U}(a)
\end{aligned}
$$

and

$$
\begin{aligned}
& (A \circ A)^{L}(1)=A^{L}(1) \wedge A^{L}(1)=A^{L}(1), \\
& (A \circ A)^{U}(1)=A^{U}(1) \wedge A^{U}(1)=A^{U}(1) .
\end{aligned}
$$

Hence $A^{2}=A$.

Example 5.9 Let $S=\{a, b, c\}$ be the semigroup with the following multiplication table:
Then $S$ is not commutative but it is regular and intraregular. We can easily see that $A \in \mathrm{IVQI}(\mathrm{S})$ for each $\tilde{0} \neq A \in D(I)^{S}$. Let $A \in \mathrm{IVQI}(\mathrm{S})$ and let $a \in S$. Then

$$
\begin{aligned}
(A \circ A)^{L}(a) & \\
= & \bigvee\left(A^{L}(x) \wedge A^{L}(y)\right) \\
= & \left(A^{L}(a) \wedge A^{L}(a)\right) \vee\left(A^{L}(a) \wedge A^{L}(b)\right) \\
& \vee\left(A^{L}(a) \wedge A^{L}(c)\right) \\
= & A^{L}(a), \\
(A \circ & A)^{U}(a)=\bigvee\left(A^{U}(x) \wedge A^{U}(y)\right) \\
= & \left(A^{U}(a) \wedge A^{U}(a)\right) \vee\left(A^{U}(a) \wedge A^{U}(b)\right) \\
& \vee\left(A^{U}(a) \wedge A^{U}(c)\right) \\
= & A^{U}(a), \\
(A \circ & A)^{L}(b)=\left(A^{L}(b) \wedge A^{L}(a)\right) \vee\left(A^{L}(b) \wedge A^{L}(b)\right) \\
& \vee\left(A^{L}(b) \wedge A^{L}(c)\right) \\
= & A^{L}(b), \\
(A \circ & A)^{U}(b)=\left(A^{U}(b) \wedge A^{U}(a)\right) \vee\left(A^{U}(b) \wedge A^{U}(b)\right) \\
& \vee\left(A^{U}(b) \wedge A^{U}(c)\right) \\
= & A^{U}(b),
\end{aligned}
$$

and

$$
\begin{aligned}
(A \circ A)^{L}(c) & \\
= & \left(A^{L}(c) \wedge A^{L}(a)\right) \vee\left(A^{L}(c) \wedge A^{L}(b)\right) \\
& \vee\left(A^{L}(c) \wedge A^{L}(c)\right) \\
= & A^{L}(c), \\
(A \circ A)^{U}(c) & \left(A^{U}(c) \wedge A^{U}(a)\right) \vee\left(A^{U}(c) \wedge A^{U}(b)\right) \\
& \vee\left(A^{U}(c) \wedge A^{U}(c)\right) \\
= & A^{U}(c) .
\end{aligned}
$$

So $A \circ A=A$. Hence each $A \in \mathrm{IVQI}(\mathrm{S})$ is idempotent.

\section{References}

[1] J. Ahsan, R. M. Latif, "Fuzzy quasi-ideals in semigroups,” J. Fuzzy Math., 9, No. 2, pp. 259-270, 2001. 
[2] A. H. Clifford, G.B.Preston, "The Algebraic Theory of Semigroup,” Vol. 1, AMS, Soc. Providence, 1961.

[3] R. Biswas, "Rosenfeld's fuzzy subgroups with interval-valued membership functions," Fuzzy Sets and Systems, 63, pp. 87-90, 1995.

[4] M. S. Cheong and K. Hur, "Interval-valued fuzzy generalized bi-ideals of a semigroup," To be submitted.

[5] J. Y. Choi, S. K. Kim and K. Hur, "Interval-valued smooth topological spaces," Honam Math. J., 32, (4), pp. 711-738, 2010.

[6] H. W. Kang and K. Hur, "Interval-valued fuzzy subgroups and subrings," Honam Math. J., 32, (4), pp. 543-617, 2010.

[7] S. Lajos, "On generalized bi-ideals in semigroups," Coll. Math. Soc. Janos Bilyai, 20, Algebraic Theory of Semigroups, North-Holland, pp. 335-340, 1979.

[8] K. C. Lee, H. W. Kang and K. Hur, "Interval-valued fuzzy ideals and bi-ideals," To be submitted.

[9] T. K. Mondal and S. K. Samanto, "Topology of interval-valued fuzzy sets," Indian J. Pure Appl. Math., 30, (1), pp. 20-38, 1999.

[10] O. Steinfeld, "Quasi-ideals in Rings and Semigroups," Akademiakiado, Budapest, 1978.

[11] L. A. Zadeh, "Fuzzy sets," Inform. and Centrol, 8, pp. 338-353, 1965.
[12] L. A. Zadeh, "The concept of a linguistic variable and its application to approximate reasoning I," Inform. Sci. 8, pp. 199-249, 1975.

\section{Sang-Mok Kim}

Associate Professor in Kwangwoon University, His research interests are Poset theory, Design theory, Combinatorial structures.

E-mail : smkim@kw.ac.kr

\section{Kul Hur}

He received B.S. degree from Yonsei University in 1972, M.S. degree from Chonbuk National University in 1974, and his Ph.D. at Yonsei University in 1987. His research interests are Category Theory, Hyperspace and Topology.

E-mail : kulhur@wonkwang.ac.kr

\section{Minseok Cheong}

Teacher in GyeongGi Science High School for the gifted, His research intersets are Poset theory, Graph theory, and Combiantorics.

E-mail : toset@hanmail.net

\section{Gab-Byung Chae}

Associate Professor in Wonkwang University, His research interests are Poset theory, Graph theory, and Combinatorics.

E-mail : rivendell@wonkwang.ac.kr 